\title{
Covariance PET patterns in early Alzheimer's disease and subjects with cognitive impairment but no dementia: utility in group discrimination and correlations with functional performance
}

\author{
Nikolaos Scarmeas, ${ }^{\mathrm{a}, \mathrm{b}, *}$ Christian G. Habeck, ${ }^{\mathrm{a}}$ Eric Zarahn, ${ }^{\mathrm{a}}$ Karen E. Anderson, ${ }^{\mathrm{c}}$ Aileen Park, ${ }^{\mathrm{a}}$ \\ John Hilton, ${ }^{a}$ Gregory H. Pelton, ${ }^{\mathrm{d}}$ Matthias H. Tabert, ${ }^{\mathrm{d}}$ Lawrence S. Honig, ${ }^{\mathrm{b}}$ James R. Moeller, ${ }^{\mathrm{d}}$ \\ Davangere P. Devanand, ${ }^{\mathrm{d}}$ and Yaakov Stern ${ }^{\mathrm{a}, \mathrm{b}, \mathrm{d}}$ \\ ${ }^{a}$ Cognitive Neuroscience Division of the Taub Institute for Research on Alzheimer's Disease and the Aging Brain, College of Physicians and Surgeons of \\ Columbia University, New York, NY 10032, USA \\ ${ }^{\mathrm{b}}$ Department of Neurology, College of Physicians and Surgeons of Columbia University, New York, NY 10032, USA \\ ${ }^{\mathrm{c}}$ Department of Psychiatry, University of Maryland, Baltimore, MD 21201, USA \\ ${ }^{\mathrm{d}}$ Department of Psychiatry, College of Physicians and Surgeons of Columbia University, New York, NY 10032, USA
}

Received 29 October 2003; revised 20 March 2004; accepted 28 April 2004

Available online 13 July 2004

\begin{abstract}
Although multivariate analytic techniques might identify diagnostic patterns that are not captured by univariate methods, they have rarely been used to study the neural correlates of Alzheimer's disease (AD) or cognitive impairment. Nonquantitative $\mathrm{H}_{2}^{15} \mathrm{O}$ PET scans were acquired during rest in 17 probable $A D$ subjects selected for mild severity [mean-modified Mini Mental Status Examination (mMMS) 46/57; SD 5.1], 16 control subjects (mMMS 54; SD 2.5) and 23 subjects with minimal to mild cognitive impairment but no dementia (mMMS 53; SD 2.8). Expert clinical reading had low success in discriminating $A D$ and controls. There were no significant mean flow differences among groups in traditional univariate SPM Voxel-wise analyses or region of interest (ROI) analyses. A covariance pattern was identified whose mean expression was significantly higher in the $\mathrm{AD}$ as compared to controls $(P=0.03$; sensitivity $76-94 \%$; specificity $63-81 \%)$. Sites of increased concomitant flow included insula, cuneus, pulvinar, lingual, fusiform, superior occipital and parahippocampal gyri, whereas decreased concomitant flow was found in cingulate, inferior parietal lobule, middle and inferior frontal, supramarginal and precentral gyri. The covariance analysis-derived pattern was then prospectively applied to the cognitively impaired subjects: as compared to subjects with Clinical Dementia Rating $(C D R)=0$, subjects with $C D R=0.5$ had significantly higher mean covariance pattern expression $(P=0.009)$. Expression of this pattern correlated inversely with Selective Reminding Test total recall $(r=-0.401, P=0.002)$, delayed recall $(r=-0.351$, $P=0.008)$ and $m M M S$ scores $(r=-0.401, P=0.002)$ in all three groups combined. We conclude that patients with $\mathrm{AD}$ may differentially express resting cerebral blood flow covariance patterns even at very early disease stages. Significant alterations in expression of resting flow covariance patterns occur even for subjects with cognitive impairment. Expression of covariance patterns correlates with cognitive and
\end{abstract}

* Corresponding author. Sergievsky Center, Columbia Presbyterian Medical Center, PH 19th floor, 622 West 168th street, New York, NY, 10032. Fax: +1-212-3421838.

E-mail address: ns257@columbia.edu (N. Scarmeas).

Available online on ScienceDirect (www.sciencedirect.com.) functional performance measures, holding promise for meaningful associations with underlying biopathological processes.

(c) 2004 Elsevier Inc. All rights reserved.

Keywords: Cognitive; Alzheimer's disease; Covariance; MCI; PET; CBF, diagnosis

Introduction

Regional cerebral blood flow (rCBF) or regional cerebral glucose metabolism (rCMRglc) deficits have been documented in patients with Alzheimer's disease (AD) either via expert clinical reading (Hoffman et al., 2000; Silverman et al., 2001) or statistical methods (usually region of interest analyses or more rarely voxelwise analyses, Ishii et al., 1997; Kennedy et al., 1995; Minoshima et al., 1997; Salmon et al., 2000; Signorini et al., 1999). Both rCBF and rCMRglc are reliable indices of neuronal/synaptic function (Jueptner and Weiller, 1995) and are considered to provide a valuable in vivo index of the effects of the neuropathological process on brain function in patients with $\mathrm{AD}$. This is supported by the fact that that reductions in $\mathrm{rCBF}$ or $\mathrm{rCMRglc}$ have been reported to co-localize with areas where AD-related neuropathological changes occur (i.e. posterior cingulate, temporal-parietaloccipital and, later in the disease, frontal association areas) (DeCarli et al., 1992; Friedland et al., 1985; Hoffman et al., 2000; McGeer et al., 1986a,b, 1990a,b; Mielke et al., 1996). Also, regions that are spared by $\mathrm{AD}$ pathology until later on in the disease (perirolandic cortex, primary visual cortex, deep structures such as basal ganglia, thalamus and cerebellum) usually manifest normal rCBF or rCMRglc (Arnold et al., 1991; DeCarli et al., 1992; Hoffman et al., 2000; Smith et al., 1992). Therefore, one of 
the explanations offered for decreased rCBF or rCMRglc is local loss of neurons and in turn decrease in the local flow and metabolic demands. Because AD is often characterized by accumulation of $\beta$ amyloid not only in the parenchyma but also in the vascular compartments, amyloid-dependent alterations in the vessel wall and resulting changes in cerebrovascular reactivity have been proposed as another possible explanation of $\mathrm{rCBF}$ reductions in AD (Gsell et al., 2000).

Since AD pathology starts from medial temporal regions (Braak and Braak, 1991), which constitute a major source of projections to medial parietal/posterior cingulate areas, it has been also hypothesized that decreased flow in parietal association areas or cingulate may be a distant effect of disruption of such projections (Aupee et al., 2001; Meguro et al., 1999; Millien et al., 2002; Vogt et al., 1992). Intrinsic cholinergic neurons of the basal forebrain (substantia innominata and nucleus basalis of Meynert) project to cortical areas and participate in the regulation of $\mathrm{rCBF}$. Because the cholinergic system of the basal forebrain, including its areas of projection to hippocampus and neocortex, are disrupted in $\mathrm{AD}$, a distant effect of cholinergic dysregulation has also been proposed as one of the possible mechanisms for reduced $\mathrm{rCBF}$ in $\mathrm{AD}$ (Gsell et al., 2000).

In addition to anatomically localized $\mathrm{rCBF}$ or rCMRglc changes, alterations in the way flow or metabolism covaries across brain areas may also occur (Grady et al., 2001). Neuropathological processes may impose a reorganization of the functional connectivity between brain areas. Because cognitive processes are the result of integrated activity in networks of areas rather than activity of any one area in isolation (Alexander and Moeller, 1994; Friston, 1994; Friston et al., 1993; Horwitz et al., 1995b; McIntosh and Gonzalez-Lima, 1994), functional connectivity can be better captured by multivariate methods.

How could connectivity at rest be conceived or interpreted? First, it is known that the brain continues to process and encode information long after external stimuli have extinguished: a cascade of biochemical processes remains active throughout a protracted consolidation period, which may last weeks in rodents and months to years in primates (Kim and Fanselow, 1992; ZolaMorgan and Squire, 1990). Therefore, rCBF changes detected at rest may be related to mnemonic cognitive processes. In addition, it has been hypothesized that large sections of the lateral and medial parietal cortex (including posterior cingulate and precuneus) and the medial frontal cortex constitute a "default network" supporting processes more active during rest than activation conditions (Lustig et al., 2003; Raichle et al., 2001). Interconnected activity of such brain areas during rest may be serving purposes of monitoring the environment, monitoring one's internal state and emotions, and various forms of undirected thought. It has been hypothesized that engagement in cognitive tasks during activation studies may redirect resources to task-specific processes and manifested as consistent deactivation of regions of this "default network".

Although some studies have used multivariate techniques during cognitive activation paradigms (Grady et al., 2001; Horwitz et al., 1995a,b; Stern et al., 2000), we know of only two studies (Alexander et al., 1994; Johnson et al., 1998) (one of which only in abstract form) which used multivariate techniques to assess changes in brain flow or metabolism at rest in AD. In contrast, these methods have been used in resting PET or SPECT studies of normal aging (Moeller et al., 1996), stroke (Leibovitch et al., 1999), epilepsy (Rubin et al., 1995), Parkinson's disease (Eidelberg et al., 1995a; Feigin et al., 2001a,b; Moeller et al., 1996), subthalamotomy, pallidotomy or pallidal stimulation in Parkinson's disease (Eidelberg et al., 1996; Fukuda et al., 2001; Su et al., 2001), Huntington's disease (Feigin et al., 2001a,b), blepharospasm (Hutchinson et al., 2000) dystonia (Eidelberg et al., 1995b, 1998) and depression (Sackeim et al., 1990, 1993).

In a few studies that have examined rCBF or rCMRglc changes in populations with cognitive deficits (at high risk for $\mathrm{AD}$ but not yet demented) (Arnaiz et al., 2001; Berent et al., 1999; De Santi et al., 2001; Minoshima et al., 1997; Okamura et al., 2002; Ouchi et al., 1998; Pietrini et al., 1993), reduced rCMRglc of the hippocampal formation (De Santi et al., 2001; Ouchi et al., 1998) has been reported. Reduced rCBF or rCMRglc in the temporal neocortex (Arnaiz et al., 2001; Berent et al., 1999; Pietrini et al., 1993) and posterior cingulate (Minoshima et al., 1997; Okamura et al., 2002) has been reported to predict conversion to AD. Only one study (Johnson et al., 1998) used multivariate techniques to examine resting flow in questionable $\mathrm{AD}$. It is conceivable that alterations in strength of functional connectivity may differ in subjects manifesting cognitive deficits secondary to insidious AD neuropathology as compared to those exhibiting benign, agerelated cognitive deficits. We hypothesized that a covariance pattern that is altered in $\mathrm{AD}$ patients (as compared to healthy elderly) would also be altered in subjects with cognitive deficits but not dementia so that it would be differentially expressed between those with more cognitive and functional deficits (presumably due to underlying $\mathrm{AD}$ ) and those with less cognitive and functional deficits (presumably due to age-related changes). We investigated this hypothesis by: (i) applying covariance analysis to PET scans from AD patients and healthy elderly, (ii) identifying a covariance pattern whose expression best discriminated the two groups, (iii) prospectively applying this derived pattern to a population with cognitive impairment (but no dementia) and (iv) investigating the relation between pattern expression and functional or cognitive measures within the population with cognitive impairment.

\section{Materials and methods}

\section{Subjects and evaluation}

Seventeen subjects with mild AD (11 males and 6 females), 23 with minimal to mild cognitive impairment but no dementia (CI) (15 males and 8 females) and 16 healthy elderly subjects ( 8 males and 8 females) met criteria for entry into the study. $\mathrm{AD}$ and $\mathrm{CI}$ subjects were recruited from outpatients who presented to the Alzheimer's Disease Research Center at Columbia University.

AD subjects met DSM-III-R criteria for dementia and NINCDSADRDA criteria for probable AD (McKhann et al., 1984). Only AD subjects rated as Clinical Dementia Rating $(C D R)=1$ (Hughes et al., 1982) were used in this study. Other causes of dementia were excluded with appropriate laboratory tests.

The CI subjects were participating in a longitudinal study, the details of which are described elsewhere (Devanand et al., 2000; Tabert et al., 2002). Inclusion criteria were the following:

(i) age $\geq 40$ years;

(ii) intellectual impairment as evidenced by either of the following:

(a) recall of two of three objects or fewer after $5 \mathrm{~min}$ on the mean-modified Mini Mental Status Examination (mMMS) (Stern et al., 1987) or a delayed recall score of $>1$ SD below normative values on the six-trial Selective Reminding Test (SRT) (Buschke and Fuld, 1974) or impaired intellectual performance as evidenced by a Wechsler Adult 
Intelligence Scale-Revised (Wechsler, 1981) performance IQ $\geq 10$ points below the verbal IQ score.

(b) subjective complaints of a decline in memory or cognitive functioning, as objectively identified by an informant and a positive score on at least one of the first eight items of the modified Blessed Functional Activity Scale (Blessed et al., 1968).

(iii) duration of intellectual impairment for $\geq 6$ months and $\leq 10$ years.

(iv) The above should result in a consensus diagnosis of "nondemented with minimal cognitive impairment" (CDR of 0 ) or “questionably demented" (CDR of 0.5) (Hughes et al., 1982).

These inclusion criteria are more inclusive as compared to those used in other studies of mild cognitive impairment (Petersen et al., 2001) because we intended to enroll a relatively broad group of subjects who presented with cognitive complaints and fell between the "normal" and "dementia" categories, whose cognitive deficits were ranging from minimal to mild severity. Based on a comprehensive review of all available clinical and diagnostic information, a consensus diagnosis by expert raters determined CDR status and study entry (Devanand et al., 2000).

The AD patients and the CI subjects underwent extensive neuropsychological evaluation including the WAIS-R (Wechsler, 1981); the mMMS (Stern et al., 1987); tests of verbal memory (shortand long-term verbal, (Buschke and Fuld, 1974); and nonverbal, (Benton, 1955; Buschke and Fuld, 1974)); orientation; abstract reasoning (verbal, (Wechsler, 1981); and nonverbal, (Mattis, 1976)); language (naming, (Kaplan et al., 1983); verbal fluency, (Benton and Hamsher, 1976; Goodglass and Kaplan, 1983); comprehension, (Goodglass and Kaplan, 1983); and repetition, (Goodglass and Kaplan, 1983)); and construction copying (Rosen, 1983); and matching, (Benton, 1955). The Blessed Dementia Rating Scale (part I, sections A and B) (Blessed et al., 1968) was administered to assess basic and instrumental activities of daily living.

Based on a comprehensive review of all available information, a consensus diagnostic conference of neurologists, psychiatrists and neuropsychologists determined the diagnosis of AD or CI, CDR status and study entry. PET results did not play any role in the diagnostic process.

Potential healthy elderly subjects were carefully screened with medical, neurological, psychiatric and neuropsychological evaluations (mMMS, (Stern et al., 1987); the American version of the Nelson Adult Reading Test estimated IQ, (Nelson and O'Connell, 1978); the WAIS-R digit symbol and vocabulary subscores, (Wechsler, 1981); and the SRT, (Buschke and Fuld, 1974)) to exclude those with dementia or cognitive impairment, as well as other neurological, psychiatric or severe medical disorders.

Subjects with any active symptoms of depression (as assessed by interviews with Neurologists and Psychiatrists) were not included in this study. Additionally, participants of this study were not receiving any central nervous system-acting medications that may affect $\mathrm{rCBF}$ or rCMRglc. Magnetic resonance imaging was used to rule out subjects with space occupying lesions or vascular disease. White matter hyperintensities were permitted but any clinical evidence (symptoms or signs) of stroke or MRI evidence of cortical or lacunar infarcts constituted exclusion criteria.

The pattern of subjects' APOE isoforms was also determined (Hixson and Powers, 1991). Due to subjects' refusal, APOE information was not available for 4/17 $\mathrm{AD}$ patients and 2/23 CI subjects. For the purpose of the analysis, subjects were categorized into three groups, those with two epsilon four alleles $(\varepsilon 4 / \varepsilon 4)$, those with one $(\varepsilon 3 / \varepsilon 4$ or $\varepsilon 2 / \varepsilon 4)$ and those without any $(\varepsilon 3 / \varepsilon 3$ or $\varepsilon 2 / \varepsilon 2$ or $\varepsilon 3 / \varepsilon 2)$.

Informed consent was obtained after the nature and risks of the study were explained. Only cross-sectional data are used for the purposes of the current analysis.

\section{PET scan acquisition and processing}

$\mathrm{H}_{2}^{15} \mathrm{O}$ resting PET scans were acquired. A bolus of $30 \mathrm{mCi}$ $\mathrm{H}_{2}^{15} \mathrm{O}$ was injected intravenously. Scan acquisition was triggered by the detection of a threshold level of true counts from the camera. Employing a Siemens EXACT 47 PET camera (Knoxville, TN, USA), two 30-s scan frames were acquired in twodimensional mode, which were subsequently averaged to yield a single image per subject. After measured attenuation correction (15-min transmission scan) and reconstruction by filtered backprojection, image resolution was $4.6 \mathrm{~mm}$ full width at halfmaximum (FWHM). Arterial blood sampling was not conducted; thus, only nonquantitative count images (and not absolute rCBF measures) could be obtained. The image of each subject was spatially transformed to the PET Montreal Neurological Institute brain space template included with SPM99 program (Wellcome Department of Neurology). The spatially transformed images were smoothed with an isotropic, Gaussian kernel (FWHM = $12 \mathrm{~mm})$.

\section{Clinical readings}

$\mathrm{AD}$ - control group discrimination was first attempted via expert clinical reading of PET scans by a dementia neurologist specialist with 10 years of experience in brain SPECT and PET evaluation who was blinded to all clinical information. A dichotomous rating for the presence or absence of the classic AD-related temporoparietal-frontal hypometabolism pattern with sparing of cerebellum, basal ganglia and sensorimotor cortex was generated. This was followed by a rating of high and low levels of confidence in the presence of the normal or $\mathrm{AD}$ pattern.

\section{Univariate analyses}

\section{Voxel-wise analyses}

The realigned, spatially transformed and smoothed PET image were intensity normalized by its average perirolandic count value. When arterial blood is not sampled, some sort of intensity normalization is typically performed (Arndt et al., 1996; Friston et al., 1990) to reduce the inter-subject and inter-session noise associated with qualitative PET. We used the average rCBF values of a region of interest corresponding to primary sensorimotor cortex as the intensity normalization factor because this region is typically spared in $\mathrm{AD}$. To effect this, a single perirolandic region of interest, constant for all subjects, was defined on the MNI template brain (using MEDX; Sensor Systems, Inc, Sterling, VA). In each subject, the average PET count value in this region of interest was determined in the spatially normalized PET image (before spatial smoothing). This value was used to divide the image values of the PET image of that subject. We reasoned that intensity normalization should reduce inter-subject scaling noise without "washing out" spatially coherent effects of AD pathology. Confirmatory SPM 
analyses were also conducted with images proportionally scaled to the global mean (the more traditional method of intensity normalization).

After normalization, voxel-wise $t$ statistics for between group (AD vs. controls, $\mathrm{AD}$ vs. $\mathrm{CI}$ and $\mathrm{CI}$ vs. controls) comparisons were computed using procedures in SPM99. The false positive rate was controlled at $\alpha_{\text {corrected }}=0.05$ per map via Bonferroni correction for the number of resolution elements (RESELS) across which regressions were calculated. Montreal Neurological Institute coordinates of local maxima of thresholded $\left(\alpha_{\text {corrected }}=0.05\right)$ SPM maps were converted to standard Talairach brain atlas (Talairach and Tournoux, 1988) coordinates (via program by Matthew Brett http:// www. mrccbu.cam.ac.uk/Imaging/mnispace.html). Using procedures in SPM99 and height thresholds corresponding to uncorrected $P$ values of 0.001 , comparisons on a cluster level were also performed.

\section{Region of interest (ROI) analyses}

$\mathrm{MED}_{\mathrm{X}}$ (Sensor Systems, Inc) software was used to overlay a standardized three-dimensional ROI template on the scan slices. This template was drawn on the SPM96 standard MRI (which conforms to Tailarach space) and defined 45 ROIs, comprising major cortical and subcortical regions. The average $\mathrm{CCBF}$ values of the ROI corresponding to primary sensory cortex (because this region is typically spared in AD) were used for scaling. Additional confirmatory ROI analyses conducted with images proportionally scaled to the cerebellum ROIs or the global mean ROI values were also performed. After normalization, $t$ statistics for between-group (AD vs. controls) comparisons of ROI rCBF values were computed. The false positive rate was controlled at $\alpha_{\text {corrected }}=0.05$ via Bonferroni correction (i.e. 45 comparisons).

\section{Covariance analysis}

Covariance analysis techniques are considered appropriate methods to explore network activity (McIntosh et al., 1996; Poline et al., 1997). We used the Scaled Subprofile Model (SSM), a type of analysis methodologically similar to other current covariance analyses techniques. SSM is a form of principal component analysis that employs the construct of "networks or patterns of localized physiological activity" to capture the multidimensional nature of functional activity at individual brain locations (Alexander and Moeller, 1994; Moeller et al., 1987). This method has been used previously in resting imaging studies of normal aging and a variety of diseases (Eidelberg et al., 1995a,b, 1996, 1998; Feigin et al., 2001a,b; Feigin et al., 2001a,b; Hutchinson et al., 2000; Moeller et al., 1996; Rubin et al., 1995; Sackeim et al., 1990, 1993; Stern et al., 2000; Su et al., 2001).

The following steps were implemented:

1. Resting voxel-wise PET data from both the AD patients and healthy elderly subjects were simultaneously included in a single SSM analysis, which captured the major sources of between- and within-group variation.

1.1. This analysis produced a series of principal components (PCs). Voxels participating in each PC may have either a positive or a negative loading. The loadings express the covariance structure (i.e. the strength of the interaction) between the voxels that participate in the PC. Voxels with positive loadings can be conceived as exhibiting con- comitant increased flow and those with negative loading can be conceived as exhibiting concomitant decreased flow. These loadings are fixed and the same for all subjects.

1.2. The expression of each $\mathrm{PC}$ for each subject was quantified by a subject scaling factor (SSF). A higher SSF value indicates more prominent concomitantly increased flow of the voxels with positive loadings and more prominent concomitantly decreased flow of the voxels with negative loadings. Therefore, the SSFs express the degree of subjects' expression of the fixed PC.

2. To identify a covariance pattern that best discriminated $A D$ patients from controls, each subject's expression (SSF) of the first four PCs derived from step 1 (capturing $31 \%$ of the variance) was entered into a linear regression model as the independent variable. Group membership (AD vs. controls) was the dependent variable. This regression resulted in a linear combination of the four PCs that best discriminated the two groups. When we use the term 'pattern' or 'network' in this manuscript, we refer to this linear combination of the first four PCs.

2.1. We used Akaike's information criterion (Burnham and Anderson, 2002) to determine how many PCs should be included in the regression to achieve optimal bias-variance trade. The first four PCs had the lowest value in Akaike's information criterion and were selected as predictors in the regression model.

2.2. The stability and robustness of weights of all voxels of the pattern were assessed via a bootstrap resampling technique (Efron and Tibshirani, 1994). Bootstrap resampling identifies areas that participate in the pattern with high confidence.

2.3. Local maxima of SPM maps were converted to standard Talairach brain atlas (Talairach and Tournoux, 1988) coordinates and labeled as described above.

3. The pattern derived in step 2 was then prospectively applied to the PET data from the CI population (which was not used for the original derivation of the pattern). The operation of forward application is represented mathematically by a "dot" product: every voxel value in a subject scan is multiplied by the corresponding voxel weight in the covariance pattern, with a subsequent summation over the whole brain volume. The resulting single number captures to what extent a subject manifests the covariance patterns that was forward applied. Using this procedure, each CI subject's SSF of the pattern was calculated.

4. Each CI subject's SSF of the pattern was entered into a linear regression model as the independent variable, with $\mathrm{CDR}$ group membership $(\mathrm{CDR}=0$ vs. $\mathrm{CDR}=0.5)$ as the dependent variable.

5. We also evaluated the correlation between SSFs for the pattern and demographic and neuropsychological performance indices. This was done across groups and in each group separately.

\section{Results}

Demographic, clinical and neuropsychological data for each of the three groups are presented in Table 1. Gender (34 males 
Table 1

Demographic, clinical and neuropsychological data for the all three groups

\begin{tabular}{|c|c|c|c|c|c|c|}
\hline & $\begin{array}{l}\text { Controls } \\
(N=16)\end{array}$ & $\begin{array}{l}\mathrm{CI} \mathrm{CDR}=0 \\
(N=14)\end{array}$ & $\begin{array}{l}\mathrm{CI} \text { CDR }=0.5 \\
(N=9)\end{array}$ & $\operatorname{AD}(N=17)$ & $\begin{array}{l}P \text { value } \\
\text { ANOVA } \\
\text { omnibus }\end{array}$ & $P$ value post hoc Tukey HSD \\
\hline Age & $71.4(6.8)$ & $64.7(9.8)$ & $73.6(6.3)$ & $68.4(8.7)$ & 0.054 & - \\
\hline Education (years) & $14.9(4.2)$ & $16.5(2.3)$ & $17.2(1.9)$ & $15.5(3.1)$ & 0.288 & - \\
\hline mMMS [0-57] & $54.1(2.5)$ & $55.3(1.6)$ & $50.8(1.8)$ & $46.4(5.1)$ & 0.000 & $\begin{array}{l}\text { AD-CI CDR 0.5: } 0.010 \\
\text { AD-CI CDR 0: } 0.000 \\
\text { AD-Controls: } 0.000 \\
\text { CI CDR 0.5-CI CDR 0: } 0.012\end{array}$ \\
\hline SRT total recall & $46.5(7.8)$ & $52.9(5.7)$ & $40.0(5.5)$ & $28.8(9.8)$ & 0.000 & $\begin{array}{l}\text { AD-CI CDR 0.5: } 0.005 \\
\text { AD-CI CDR 0: } 0.000 \\
\text { AD-Controls: } 0.000 \\
\text { CI CDR 0.5-CI CDR 0: } 0.002\end{array}$ \\
\hline SRT delayed recall & $7(3.2)$ & $8.7(2.6)$ & $3.9(2.0)$ & $2.0(2.2)$ & 0.000 & $\begin{array}{l}\text { AD-CI CDR 0: } 0.000 \\
\text { AD-Controls: } 0.000 \\
\text { CI CDR 0.5-CI CDR 0: } 0.002 \\
\text { CI CDR } 0.5 \text {-Controls: } 0.033\end{array}$ \\
\hline Digit symbol & $12.5(2.3)$ & $13.6(3.2)$ & $10.9(2.1)$ & $9.7(3.5)$ & 0.002 & $\begin{array}{l}\mathrm{AD}-\mathrm{CI} \text { CDR } 0: 0.002 \\
\mathrm{AD}-\text { Controls } 0.031\end{array}$ \\
\hline
\end{tabular}

Mean values and standard deviations (in parentheses) are reported. Initial $P$ values are calculated with one-way ANOVA. Significant $P$ values derived from post hoc Tukey HSD analyses are also reported.

and 22 females) did not differ across groups $(P=0.65)$. CI $\mathrm{CDR}=0$ subjects were slightly younger than $\mathrm{CI} \mathrm{CDR}=0.5$. Education did not differ across groups. Neuropsychological performance in the $\mathrm{AD}$ patients was significantly lower than that of controls or $\mathrm{CI} C D R=0$ groups for all tests. CI CDR $=$ 0.5 subjects performed significantly better than $\mathrm{AD}$ patients on the mMMS and SRT total recall, while their performance on SRT delayed recall and digit symbol tests was comparable to the $\mathrm{AD}$ patients. CI subjects with $\mathrm{CDR}=0.5$ performed worse than $\mathrm{CI} C D R=0$ on mMMS and all SRT measures, and worse than controls on SRT delayed recall.

Expert blinded clinical reading of this set of early $\mathrm{AD}$ and control $\mathrm{H}_{2}^{15} \mathrm{O}$ PET scans had low success in classifying group membership: sensitivity was $54 \%$ and specificity $25 \%$.

The univariate voxel-wise comparisons did not reveal any between-group differences (irrespective of whether images were proportionally scaled to perirolandic cortex or global mean). The groups did not differ on the cluster-level comparisons either. These was true for $\mathrm{AD}$-control, $\mathrm{AD}-\mathrm{CI}$ and $\mathrm{CI}-$ control comparisons.

In the univariate ROI analyses, $\mathrm{rCBF}$ in the left inferior parietal ROI was significantly lower in the $\mathrm{AD}$ patients as compared to controls $(P=0.04)$. Left posterior cingulate ROI $(P=0.08)$ and left hippocampus ROI $(P=0.10)$ had also somehow lower $\mathrm{rCBF}$ for the $\mathrm{AD}$ patients. However, after controlling for the false positive rate, the differences were not significant for any of the regions. Additional confirmatory ROI analyses conducted with images proportionally scaled to the cerebellum ROIs or the global mean ROI values did not produce any different results.

The pattern identified with the multivariate method is presented in Fig. 1. Positive loadings (concomitant increased flow) were noted in bilateral insula, lingual gyri and cuneus; left fusiform and superior occipital gyri; and right parahippocampal gyrus and pulvinar (Table 2). Negative loadings (concomitant decreased flow) were noted in bilateral inferior parietal lobule and cingulate; and left middle frontal, inferior frontal, precentral and supramarginal gyri (Table 3).

Mean expression of the pattern was higher in the AD group than in the healthy elderly $(P=0.03)$ (Fig. 2A). Thus, compared to the healthy elders, AD subjects had more concomitantly increased flow at areas with positive loading and more prominent concomitantly decreased flow at areas with negative loadings. An ROC curve was used to determine an SSF cutoff that produced optimal sensitivity and specificity. At a SSF cutoff of 19.5 , the pattern was $76 \%$ sensitive and $81 \%$ specific for discriminating AD vs. control. At a cutoff of 15.7 , sensitivity was $94 \%$ and specificity $63 \%$. When age was included as covariate the results were unchanged.

When the pattern derived from the control and AD group was prospectively applied to the CI group, expression of the pattern was higher in the $9 \mathrm{CI}$ subjects with $\mathrm{CDR}=0.5$ as compared to

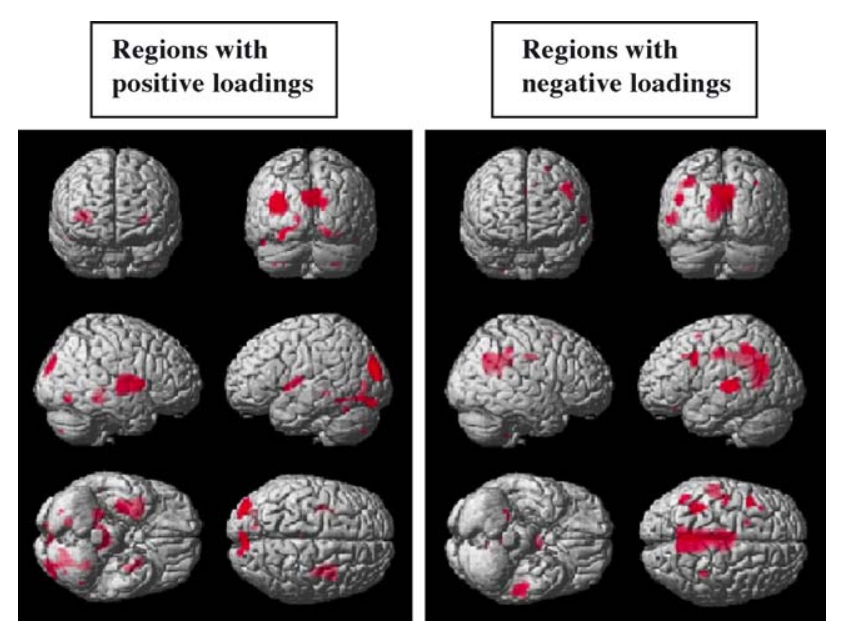

Fig. 1. Three-dimensional brain rendering representation of statistical parametric map of the 'AD-control pattern'. 
Table 2

Talairach coordinates of areas in discriminatory covariance pattern with positive loadings

\begin{tabular}{rlrll}
\hline $\mathrm{X}$ & $\mathrm{Y}$ & $\mathrm{Z}$ & Location & $\begin{array}{l}\text { Brodmann } \\
\text { area }\end{array}$ \\
\hline 36 & -11 & 4 & right insula & 13 \\
-48 & -55 & -14 & left fusiform gyrus & 37 \\
-16 & -74 & -1 & left lingual gyrus & 18 \\
-24 & -70 & -5 & left lingual gyrus & 19 \\
-14 & -74 & 6 & left cuneus & 18 \\
12 & -37 & -2 & right parahippocampal gyrus & 30 \\
8 & -31 & 0 & right pulvinar & - \\
-32 & -5 & 9 & left insula & - \\
0 & -84 & 32 & left cuneus & 19 \\
12 & -88 & 27 & right cuneus & 19 \\
16 & -70 & -7 & right lingual gyrus & 18 \\
-34 & -84 & 28 & left superior occipital gyrus & 19 \\
\hline
\end{tabular}

The areas are presented in order of decreasing but still fulfilling significance criterion for bootstrap (ICV $>2, P<0.03$ ) contribution to the pattern.

the 14 CI subjects with $\mathrm{CDR}=0(P=0.009)$ (Fig. 2A). Thus, CI subjects with greater functional deficits expressed the pattern more like the AD group, while those CI subjects without functional deficits expressed the pattern more like the healthy elderly control group. Because of slight age difference between the two CI groups, we controlled for age in a supplementary analysis: the results were unchanged.

Table 4 presents correlations of expression of the pattern (each subject's SSF) with demographic and behavioral variables within each of the three groups and for all subjects combined. Expression of the pattern did not correlate with age, sex, education or APOE status. Expression of the pattern correlated negatively with SRT total recall score and mMMS in the CI group (Table 4 and Fig. 2B). When all subjects were pooled, there was also a negative correlation between pattern expression and SRT total recall, delayed recall and mMMS scores. These results are in accordance with the increasing mean pattern expression in the four subject groups (controls $<$ CI-CDR $=0$ $<$ CI-CDR $=0.5<\mathrm{AD}$ ): expression increased with the severity of functional deficit (Fig. 2A). Thus, increasing expression of the

Table 3

Talairach coordinates of areas in discriminatory covariance pattern with negative loadings

\begin{tabular}{rrrlr}
\hline X & Y & Z & Location & $\begin{array}{c}\text { Brodmann } \\
\text { area }\end{array}$ \\
\hline-8 & -14 & 38 & cingulate & 24 \\
2 & -55 & 27 & cingulate & 31 \\
-40 & 12 & 36 & left middle frontal gyrus & 9 \\
-46 & 11 & 27 & left inferior frontal gyrus & 9 \\
-36 & 23 & 34 & left precentral gyrus & 9 \\
-32 & 28 & -18 & left inferior frontal gyrus & 47 \\
-48 & -53 & 28 & left supramarginal gyrus & 40 \\
-32 & -41 & 43 & left inferior parietal lobule & 40 \\
-44 & -12 & 39 & left precentral gyrus & 4 \\
-4 & -37 & 30 & cingulate & 31 \\
6 & -41 & 26 & cingulate & 23 \\
34 & -37 & 41 & right inferior parietal lobule & 40 \\
\hline
\end{tabular}

The areas are presented in order of decreasing but still fulfilling significance criterion for bootstrap (ICV $<-2, P<0.03$ ) contribution to the pattern.

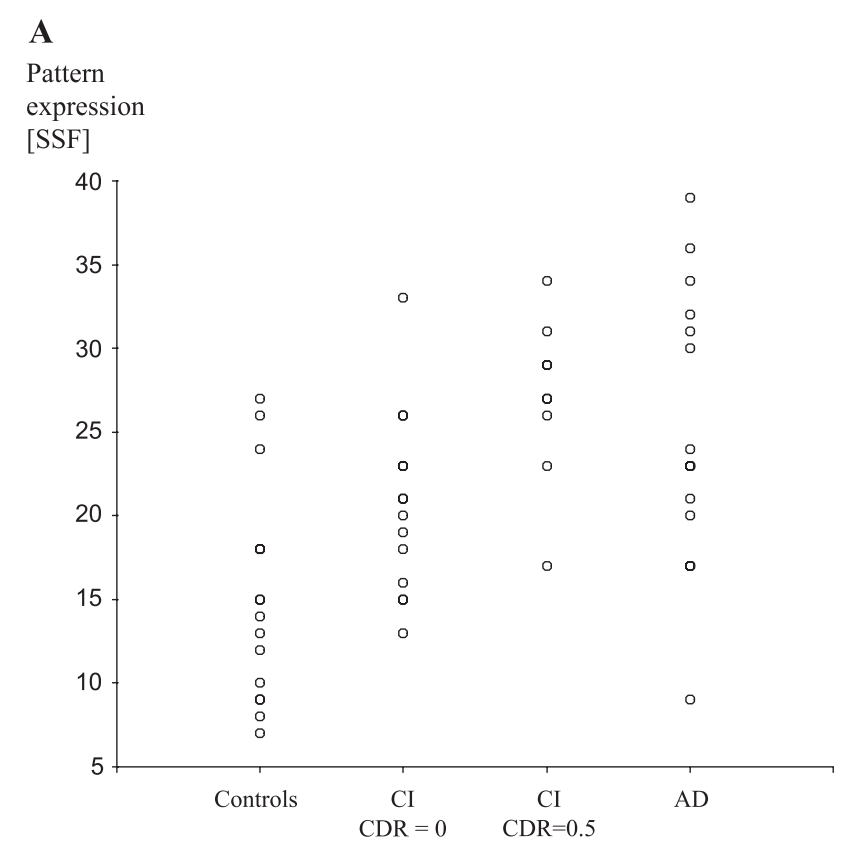

B

Pattern

expression

[SSF]

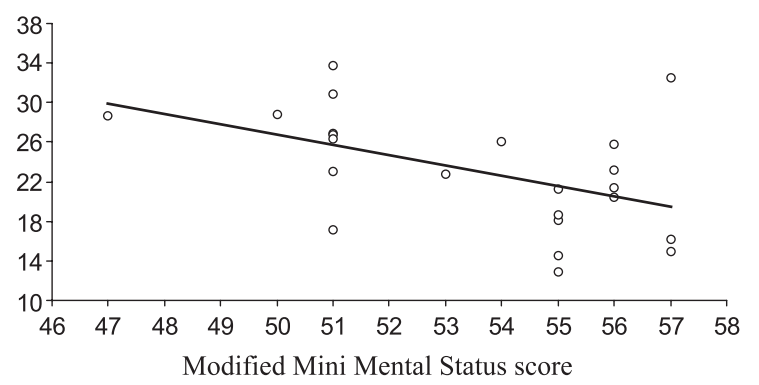

Pattern

expression

[SSF]

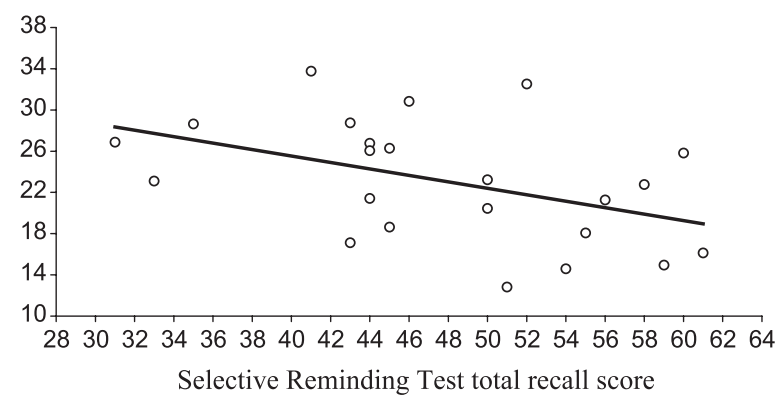

Fig. 2. (A) Expression of the pattern in the controls and AD patients, and expression derived from prospective application of the pattern to CI with $\mathrm{CDR}=0$ and $\mathrm{CI}$ with $\mathrm{CDR}=0.5$. Note the gradual increase in pattern expression in groups with gradually increasing functional impairment. (B) Expression of the pattern plotted against mMMS score and SRT total recall score for the CI group only (correlation coefficients and significance values are presented in Table 4). Although the pattern is derived from the AD and control population, note the association between its expression and cognitive performance measures when prospectively applied in the CI group. 
Table 4

Pearson correlation coefficients $(r)$ between expressions of the pattern (each subject's SSF) and demographic and behavioral variables within each of the three groups and for all subjects combined

\begin{tabular}{|c|c|c|c|c|}
\hline & Controls $(N=16)$ & $\mathrm{CI}(N=23)$ & $\operatorname{AD}(N=17)$ & All subjects $(N=56)$ \\
\hline Age & $-0.106(0.697)$ & $0.372(0.081)$ & $-0.197(0.449)$ & $-0.035(0.797)$ \\
\hline Gender & $-0.010(0.970)$ & $-0.157(0.475)$ & $0.337(0.186)$ & $-0.020(0.887)$ \\
\hline Education (years) & $-0.050(0.853)$ & $0.244(0.262)$ & $0.413(0.099)$ & $0.240(0.074)$ \\
\hline APOE genotype* & $-0.197(0.465)$ & $0.032(0.891)$ & $0.423(0.150)$ & $0.171(0.236)$ \\
\hline mMMS [0-57] & $0.188(0.485)$ & $-\mathbf{0 . 5 0 2}(0.015)$ & $-0.362(0.153)$ & $-\mathbf{0 . 4 0 1}(0.002)$ \\
\hline Selective Reminding Test total recall & $-0.266(0.320)$ & $-0.444(0.034)$ & $-0.337(0.185)$ & $-\mathbf{0 . 4 0 1}(0.002)$ \\
\hline Selective Reminding Test delayed recall & $-0.276(0.301)$ & $-0.304(0.158)$ & $-0.165(0.526)$ & $-\mathbf{0 . 3 5 1}(0.008)$ \\
\hline Digit symbol (age adjusted) & $-0.129(0.634)$ & $0.004(0.987)$ & $-0.303(0.254)$ & $-0.231(0.090)$ \\
\hline
\end{tabular}

$P$ values are reported in parentheses. Significant correlation coefficients are reported in bold format.

* APOE information was available for all 16/16 Controls, 21/23 CI subjects and 13/17 AD patients (50/56 subjects overall).

pattern was accompanied by decreasing memory and cognitive performance (Table 4).

\section{Discussion}

Expert clinical reading and univariate analyses could not discriminate resting scans of mild $\mathrm{AD}$ patients and controls. However, expression of a PET covariance pattern was significantly altered in the AD patients (as compared to controls). When this pattern was prospectively applied to a CI population, its expression significantly differed among CI subjects with and without functional deficits. Those with more deficits expressed the pattern more like AD patients. Expression of this pattern also correlated with neuropsychological performance.

\section{AD-control discrimination}

FDG PET clinical reading using AD subjects with all levels of clinical severity has an approximate sensitivity of $93 \%$ with specificity of $63-76 \%$ (Hoffman et al., 2000; Silverman et al., 2001). In the literature of voxel-wise analyses, reduced $\mathrm{rCBF}$ in temporal, parietal and cingulate cortex has been noted in $\mathrm{AD}$ patients in comparison to controls (Ishii et al., 1997; Kennedy et al., 1995; Minoshima et al., 1997; Salmon et al., 2000; Signorini et al., 1999). With the exception of one study (Ishii et al., 1997), all other previous reports included moderate- or even advanced-stage AD patients (Kennedy et al., 1995; Minoshima et al., 1997; Salmon et al., 2000; Signorini et al., 1999). It is also unclear whether all of these studies used corrections for multiple comparisons in their statistical tests.

Because it was conceivable that voxel-wise analysis may have resulted in low signal-to-noise ratio, we also explored our data with ROI analyses. Overall, in this data set, the AD-control differences were small: they did not survive the multiple comparison correction in the ROI analysis and they could not be visually identified via expert clinical reading. Probably the most important reason for failure of expert clinical reading or univariate analyses to demonstrate significant differences between $\mathrm{AD}$ patients and controls in the current study was the exclusive recruitment of mild AD patients. This could have minimized rCBF changes in comparison to controls. However, it is notable that the ROI analysis identified a tendency for decreased $\mathrm{rCBF}$ at parietal, posterior cingulate and hippocampal regions. Since these are the areas where early AD pathological changes and $\mathrm{rCBF}$ reductions are usually noted, the ROI analyses provide reassurance for the quality of the data and the methodology used.
A multivariate method that concentrated on the covariance of rCBF across brain areas proved to be a relatively more powerful tool with sensitivity $76-94 \%$ and specificity $63-81 \%$. A previous study had reported a diagnostic accuracy of $93.4 \%$ in discriminating between $\mathrm{AD}$ patients and controls with use of multivariate techniques (Johnson et al., 1998). Nevertheless, there was still considerable overlap in pattern expression among AD patients and controls; therefore, the derived pattern cannot qualify as a sufficient diagnostic test in clinical settings.

\section{CI subjects}

When the pattern that was derived from the controls and $\mathrm{AD}$ patients was prospectively applied to the subjects with CI, the degree of each subject's expression of the pattern was different for subjects with $\mathrm{CDR}=0$ as compared to the ones with $\mathrm{CDR}=0.5$. Expression of the pattern in the CI group also correlated with neuropsychological test scores. Given that CI subjects who express the pattern to a greater degree demonstrated functional deficits and that $\mathrm{AD}$ subjects expressed the pattern to an even greater degree, we hypothesize that higher pattern expression may be an indicator for insidious AD pathology. Without prospective follow-up, we can only speculate that CI with higher levels of topographic expression are at a greater risk of conversion to AD. One previous study used multivariate techniques in analyzing brain Tc-HMPAO SPECT data from a questionable AD population (Johnson et al., 1998). In that study, the accuracy of discriminating 27 questionable $\mathrm{AD}$ subjects who did not progress from 18 questionable AD subjects who converted to AD over a 2-year follow-up was $84.4 \%$. The current study is limited to strictly cross-sectional information.

The most important advantage of the current analysis is that the pattern was derived from the $\mathrm{AD}$-control comparison and was then prospectively applied to the CI PET data. The fact that expression of the identified pattern correlated with functional and cognitive measures in a population whose PET data were not used to derive the pattern provides strong evidence for its validity and generalizability. Our success in reproducing pattern expression in a new population is in accordance with previous reports (Moeller et al., 1996).

\section{Pattern expression and cognitive measures}

Although CDR ratings were derived via a consensus diagnostic conference, they may contain subjective elements. Thus, it is notable that pattern expression also correlated with cognitive performance as objectively measured by neuropsychological test- 
ing (Table 4). Notably, the association between pattern expression and neuropsychological performance remained significant when evaluated in the CI group alone (Table 4 and Fig. 2B). The directionality of the associations was consistent with the increasing mean pattern expression across the four groups (controls $<$ CI$\mathrm{CDR}=0<\mathrm{CI}-\mathrm{CDR}=0.5<\mathrm{AD}$ ): increasing expression of the pattern was accompanied by increasing cognitive severity of deficit. The correlations were not significant in the control or $\mathrm{AD}$ groups alone, probably because of restricted variability in neuropsychological performance and loss of power.

\section{Single vs. multiple covariance patterns}

Our analyses derived one pattern, which was expressed by all subjects. The correlation structure between $\mathrm{rCBF}$ of brain areas that participate in this pattern is fixed and the same for all subjects. What differed among groups (and correlated with cognitive and functional performance) was each subject's degree of expression of this pattern. The degree of expression of the covariance pattern directly addresses how flow in different brain areas is modulated as a function of disease status, while keeping the strength of the interaction between areas constant. This might imply that AD pathology affected the degree of pattern expression without destroying the covariance pattern itself. It might also be possible to identify different patterns for controls and AD patients. This would imply that AD pathology impaired one pattern, which was in turn replaced with a completely different new one.

We did not explore this alternate possibility. If separate patterns were derived (one for the $\mathrm{AD}$ and a different one for the control group), their expression would relate mostly to separate withingroup variances and not directly address between-group differences. Our strategy for identification of a single pattern is a more conservative approach. It has the advantage of a direct comparison of the two groups along one dimension, that is, with respect to the subject expression of one single pattern. In addition, deriving a single pattern provides the opportunity to prospectively apply the pattern to the CI group. The obtained levels of expression in the CI group then has a straightforward interpretation relative to the expression in the other two groups: for some of the CI subjects, the degree of pattern expression is more like the AD patients and for some others more like the controls.

\section{Multivariate analyses of activation studies in $A D$}

Several groups have used multivariate methods in cognitive activation paradigms to investigate the way in which neuropathological processes may impose a reorganization of brain networks. In one study, path analysis indicated an altered functional network in mild $\mathrm{AD}$ patients (in comparison to controls) during a facematching task (Horwitz et al., 1995b). Another study indicated that PET data from healthy elderly demonstrated a strong functional linkage along a right hemisphere ventral network (including occipital, temporal, and frontal regions), which was similar to young subjects. However, AD patients did not use the same functional network (Horwitz et al., 1995a) despite their ability to perform the task with the same accuracy.

In a delayed match to sample short-term memory paradigm administered in early AD patients and healthy elderly (Grady et al., 2001), memory breakdown in AD was shown to be related to functional disconnection within a distributed network that included the prefrontal cortex and the hippocampus. In addition, a possible compensatory role for an amygdala-emotion related network was suggested for the AD patients.

In another cognitive activation study, multivariate techniques were utilized to compare brain $\mathrm{H}_{2}^{15} \mathrm{O}$ PET activities between early $\mathrm{AD}$ and healthy elders during performance of a serial verbal recognition task (Stern et al., 2000). While healthy elders recruited a network involving left anterior cingulate and anterior insula, AD patients recruited an alternative network involving left posterior temporal cortex, calcarine cortex, posterior cingulate and the vermis.

\section{Multivariate analyses, resting data and biopathological considerations}

Imaging CBF changes during activation studies typically reflect acute changes in bioelectric phenomena (action potentials and postsynaptic potentials) (Logothetis et al., 1999) while resting flow changes (which are tightly coupled with glucose metabolism changes) reflect a wide range of biochemical processes including all the molecular machinery required for normal neuronal function (signal transduction, second messenger cascades, protein synthesis, axonal transport, but most of all synaptic activity) (Erecinska and Silver, 1989; Jueptner and Weiller, 1995). Multivariate techniques have been used to explore resting imaging data in many neurological and psychiatric conditions (Eidelberg et al., 1995a,b, 1996, 1998; Feigin et al., 2001a,b; Feigin et al., 2001a,b; Fukuda et al., 2001; Hutchinson et al., 2000; Moeller et al., 1996; Nakamura et al., 2001; Rubin et al., 1995; Sackeim et al., 1990, 1993; Su et al., 2001). RCBF covariance among brain regions that participate in the pattern may be thought of as coordinated biochemical or synaptic activity, suggesting some sort of connectivity between these areas.

The fact that (i) expression of the identified covariance pattern differed between $\mathrm{AD}$ and controls and that (ii) higher expression of the pattern was associated with worse memory suggests that the degree of expression of this pattern is related to disease status.

It has been hypothesized that interconnected activity of brain areas such as lateral and medial parietal cortex (including posterior cingulate and precuneus) and the medial frontal cortex during rest may be constitute a "default network" serving purposes of monitoring the environment, monitoring one's internal state and emotions, and various forms of undirected thought (Lustig et al., 2003; Raichle et al., 2001). Interestingly, many of these regions that exhibit high metabolism during rest at young people show reduced resting metabolism in elderly, and even more reduction in AD (Herholz et al., 2002). In accordance to these observations, we noted that, as compared to healthy elderly, AD patients had more prominent concomitantly decreased flow in areas of this "default network" [such as medial (cingulate) and lateral (supramarginal gyrus and inferior parietal lobule) parietal].

The concomitantly decreased flow in these areas may be a distant effect of AD-related changes of entorhinal/perirhinal cortex. Medial temporal regions are a major source of projections to medial parietal/posterior cingulate areas and their disruption leads to hypometabolism in humans and animals (Aupee et al., 2001; Meguro et al., 1999; Millien et al., 2002; Vogt et al., 1992). Alternatively, it is also possible that we are observing the direct effect of $\mathrm{AD}$ pathology itself: decreased $\mathrm{rCBF}$ or rCMRglc has been reported to co-localize with areas where $\mathrm{AD}$-related neuropathological changes occur and is therefore considered to represent an in vivo index of the effect of the neuropathological 
process on brain function. The cingulate, inferior parietal lobule and supramarginal gyrus, which are pathologically affected and manifest decreased $\mathrm{rCBF}$ or rCMRglc early in $\mathrm{AD}$ in univariate analyses (Hoffman et al., 2000; Ishii et al., 1997; Kennedy et al., 1995; Minoshima et al., 1997; Silverman et al., 2001), also showed concomitant decreased $\mathrm{rCBF}$ in our study. However, medial temporal areas like the parahippocampal gyrus, which is also pathologically affected and manifests decreased $\mathrm{rCBF}$ or rCMRglc early in AD in univariate analyses, exhibited concomitant increased flow in our covariance pattern. Areas participating in the covariance patterns are somehow interconnected, that is, changes in one area affect other areas of the pattern. The presence of the pattern even at a healthy stage suggests that cingulateinferior parietal lobule-supramarginal gyrus on one hand and parahippocampal gyrus on the other are interconnected such that they manifest concomitant flow of opposite directionality. In AD patients the connectivity-covariance among these areas is present (the pattern is still expressed) but it is more exaggerated in degree (higher SSFs). It may be the case that reduced flow because of AD pathology in certain nodes of the pattern (cingulate, inferior parietal lobule, supramarginal gyrus) affects the whole pattern and results in relatively increased flow in a different node (parahippocampal gyrus). This in accordance with a recent report indicating increased hippocampal region $\mathrm{rCBF}$ (assessed by arterial spin labeling perfusion MRI) in early $\mathrm{AD}$, which gradually subsided as AD became more severe (Press et al., 2003). In that study, up-regulation of flow in hippocampal region was contrasted to marked rCBF decrease in neocortex and was interpreted as a possible compensatory response in the hippocampal region.

Similarly, brain areas participating in covariance patterns need not include only areas affected by neuropathology. For example, the precentral gyrus (known to be spared by AD pathology) participates in the observed pattern along with areas that may be affected by the disease (like the cingulate and parietal areas). Again, more prominent decreased flow in this area for the AD patients may be the result of a perturbation in other nodes of the network (i.e. AD pathology in cingulate, inferior parietal lobule and supramarginal gyrus).

The differences in degree of pattern expression between $\mathrm{AD}$ patients and controls are interesting from a biological point of view. It suggests that some areas are interconnected in healthy elders. When some of these areas are affected by AD pathology, then relative concomitant flow or synaptic activity in other areas of this network is also affected. In addition, this network perturbation seems to occur early in the course of the disease, perhaps even during its preclinical stages.

We sought to investigate cerebral covariance patterns in AD and subjects with cognitive impairment using resting PET data. We used established multivariate methods that have been shown useful in cognitive activation imaging studies of healthy subjects, cognitive activation studies of AD patients and resting imaging studies of other neurological and psychiatric diseases. These methods provide information about the way that brain areas covary with each other that is not captured by traditional univariate analyses. Although diagnostic properties of covariance patterns are still limited for use in everyday clinical practice on a person by person basis, alterations in expression of these patterns may be a sensitive index of cerebral flow changes as a result of AD pathology even at early stages. The reproduction and properties of these patterns in our CI population suggesting meaningful associations with under- lying biopathological processes holds promise for identification of subgroups of subjects with cognitive impairment with different etiology and prognosis.

\section{Acknowledgments}

This research was supported by Federal Grants AG 14671, AG 17761, RR 00645 and the Alzheimer's Association.

\section{References}

Alexander, G.E., Moeller, J.R., 1994. Application of the scaled subprofile model to functional imaging in neuropsychiatric disorders: a principal component approach. Hum. Brain Mapp. 2 (1-2), 79-94.

Alexander, G.E., Moeller, J.R., Grady, C.L., Pietrini, P., Mentis, M.J., Schapiro, M.B., 1994. Association of cognitive functions with regional networks of brain metabolism in Alzheimer-disease. Neurobiol. Aging 15 (Suppl. 1), s36.

Arnaiz, E., Jelic, V., Almkvist, O., Wahlund, L.O., Winblad, B., Valind, S., Nordberg, A., 2001. Impaired cerebral glucose metabolism and cognitive functioning predict deterioration in mild cognitive impairment. NeuroReport 12 (4), 851-855.

Arndt, S., Cizadlo, T., O’Leary, D., Gold, S., Andreasen, N.C., 1996. Normalizing counts and cerebral blood flow intensity in functional imaging studies of the human brain. NeuroImage 3 (3 Pt 1), 175-184.

Arnold, S.E., Hyman, B.T., Flory, J., Damasio, A.R., Van Hoesen, G.W., 1991. The topographical and neuroanatomical distribution of neurofibrillary tangles and neuritic plaques in the cerebral cortex of patients with Alzheimer's disease. Cereb. Cortex 1 (1), 103-116.

Aupee, A.M., Desgranges, B., Eustache, F., Lalevee, C., de la Sayette, V., Viader, F., Baron, J.C., 2001. Voxel-based mapping of brain hypometabolism in permanent amnesia with PET. NeuroImage 13 (6 Pt 1), $1164-1173$.

Benton, A., 1955. The Visual Retention Test. The Psychological Corp., New York.

Benton, A., Hamsher, A., 1976. Multiligual Aphasia Examination. University of Iowa, Iowa City.

Berent, S., Giordani, B., Foster, N., Minoshima, S., Lajiness-O’Neill, R., Koeppe, R., Kuhl, D.E., 1999. Neuropsychological function and cerebral glucose utilization in isolated memory impairment and Alzheimer's disease. J. Psychiatr. Res. 33 (1), 7-16.

Blessed, G., Tomlinson, B.E., Roth, M., 1968. The association between quantitative measures of dementia and of senile change in the cerebral grey matter of elderly subjects. Br. J. Psychiatry 114 (512), 797-811.

Braak, H., Braak, E., 1991. Neuropathological stageing of Alzheimer-related changes. Acta Neuropathol. (Berl.) 82 (4), 239-259.

Burnham, K.P., Anderson, D.R., 2002. Model Selection and Multimodel Inference Springer Verlag, New York.

Buschke, H., Fuld, P.A., 1974. Evaluating storage, retention, and retrieval in disordered memory and learning. Neurology 24 (11), 1019-1025.

DeCarli, C., Atack, J.R., Ball, M.J., Kay, J.A., Grady, C.L., Fewster, P., Pettigrew, K.D., Rapoport, S.I., Schapiro, M.B., 1992. Post-mortem regional neurofibrillary tangle densities but not senile plaque densities are related to regional cerebral metabolic rates for glucose during life in Alzheimer's disease patients. Neurodegeneration 1, 113-121.

De Santi, S., de Leon, M.J., Rusinek, H., Convit, A., Tarshish, C.Y., Roche, A., Tsui, W.H., Kandil, E., Boppana, M., Daisley, K., Wang, G.J., Schlyer, D., Fowler, J., 2001. Hippocampal formation glucose metabolism and volume losses in MCI and AD. Neurobiol. Aging 22 (4), 529-539.

Devanand, D.P., Michaels-Marston, K.S., Liu, X., Pelton, G.H., Padilla, M., Marder, K., Bell, K., Stern, Y., Mayeux, R., 2000. Olfactory deficits in patients with mild cognitive impairment predict Alzheimer's disease at follow-up. Am. J. Psychiatry 157 (9), 1399-1405. 
Efron, B., Tibshirani, R.J., 1994. An Introduction to the Bootstrap. CRC Press, LLC, New York.

Eidelberg, D., Moeller, J.R., Ishikawa, T., Dhawan, V., Spetsieris, P., Chaly, T., Belakhlef, A., Mandel, F., Przedborski, S., Fahn, S., 1995a. Early differential diagnosis of Parkinson's disease with 18Ffluorodeoxyglucose and positron emission tomography. Neurology 45 (11), 1995-2004

Eidelberg, D., Moeller, J.R., Ishikawa, T., Dhawan, V., Spetsieris, P., Przedborski, S., Fahn, S., 1995b. The metabolic topography of idiopathic torsion dystonia. Brain 118 (Pt 6), 1473-1484.

Eidelberg, D., Moeller, J.R., Ishikawa, T., Dhawan, V., Spetsieris, P., Silbersweig, D., Stern, E., Woods, R.P., Fazzini, E., Dogali, M., Beric, A., 1996. Regional metabolic correlates of surgical outcome following unilateral pallidotomy for Parkinson's disease. Ann. Neurol. 39 (4), $450-459$.

Eidelberg, D., Moeller, J.R., Antonini, A., Kazumata, K., Nakamura, T., Dhawan, V., Spetsieris, P., deLeon, D., Bressman, S.B., Fahn, S., 1998. Functional brain networks in DYT1 dystonia. Ann. Neurol. 44 (3), $303-312$

Erecinska, M., Silver, I.A., 1989. ATP and brain function. J. Cereb. Blood Flow Metab. 9 (1), 2-19.

Feigin, A., Fukuda, M., Dhawan, V., Przedborski, S., Jackson-Lewis, V., Mentis, M.J., Moeller, J.R., Eidelberg, D., 2001a. Metabolic correlates of levodopa response in Parkinson's disease. Neurology 57 (11), $2083-2088$.

Feigin, A., Leenders, K.L., Moeller, J.R., Missimer, J., Kuenig, G., Spetsieris, P., Antonini, A., Eidelberg, D., 2001b. Metabolic network abnormalities in early Huntington's disease: an [18F]FDG PET study. J. Nucl. Med. 42 (11), 1591-1595.

Friedland, R.P., Brun, A., Budinger, T.F., 1985. Pathological and positron emission tomographic correlations in Alzheimer's disease. Lancet 1 (8422), 228.

Friston, K.J., 1994. Functional and effective connectivity in neuroimaging: a synthesis. Hum. Brain Mapp. 2 (1-2), 56-78.

Friston, K.J., Frith, C.D., Liddle, P.F., Dolan, R.J., Lammertsma, A.A., Frackowiak, R.S., 1990. The relationship between global and local changes in PET scans. J. Cereb. Blood Flow Metab. 10 (4), 458-466.

Friston, K.J., Frith, C.D., Liddle, P.F., Frackowiak, R.S., 1993. Functional connectivity: the principal-component analysis of large (PET) data sets. J. Cereb. Blood Flow Metab. 13 (1), 5-14.

Fukuda, M., Mentis, M.J., Ma, Y., Dhawan, V., Antonini, A., Lang, A.E., Lozano, A.M., Hammerstad, J., Lyons, K., Koller, W.C., Moeller, J.R., Eidelberg, D., 2001. Networks mediating the clinical effects of pallidal brain stimulation for Parkinson's disease: a PET study of resting-state glucose metabolism. Brain 124 (Pt 8), 1601-1609.

Goodglass, H., Kaplan, D., 1983. The assessment of Aphasia and Related Disorders Lea \& Febiger, Philadelphia.

Grady, C.L., Furey, M.L., Pietrini, P., Horwitz, B., Rapoport, S.I., 2001. Altered brain functional connectivity and impaired short-term memory in Alzheimer's disease. Brain 124 (Pt 4), 739-756.

Gsell, W., De Sadeleer, C., Marchalant, Y., MacKenzie, E.T., Schumann, P., Dauphin, F., 2000. The use of cerebral blood flow as an index of neuronal activity in functional neuroimaging: experimental and pathophysiological considerations. J. Chem. Neuroanat. 20 (3-4), 215-224.

Herholz, K., Salmon, E., Perani, D., Baron, J.C., Holthoff, V., Frolich, L., Schonknecht, P., Ito, K., Mielke, R., Kalbe, E., Zundorf, G., Delbeuck, X., Pelati, O., Anchisi, D., Fazio, F., Kerrouche, N., Desgranges, B., Eustache, F., Beuthien-Baumann, B., Menzel, C., Schroder, J., Kato, T., Arahata, Y., Henze, M., Heiss, W.D., 2002. Discrimination between Alzheimer dementia and controls by automated analysis of multicenter FDG PET. NeuroImage 17 (1), 302-316.

Hixson, J.E., Powers, P.K., 1991. Restriction isotyping of human apolipoprotein A-IV: rapid typing of known isoforms and detection of a new isoform that deletes a conserved repeat. J. Lipid Res. 32 (9), $1529-1535$.

Hoffman, J.M., Welsh-Bohmer, K.A., Hanson, M., Crain, B., Hulette, C.,
Earl, N., Coleman, R.E., 2000. FDG PET imaging in patients with pathologically verified dementia. J. Nucl. Med. 41 (11), 1920-1928.

Horwitz, B., McIntosh, A.R., Haxby, J.V., Furey, M., Salerno, J.A., Schapiro, M.B., Rapoport, S.I., Grady, C.L., 1995a. Network analysis of PET-mapped visual pathways in Alzheimer type dementia. NeuroReport 6 (17), 2287-2292.

Horwitz, B., McIntosh, A.R., Haxby, J.V., Grady, C.L., 1995b. Network analysis of brain cognitive function using metabolic and blood flow data. Behav. Brain Res. 66 (1-2), 187-193.

Hughes, C.P., Berg, L., Danziger, W.L., Coben, L.A., Martin, R.L., 1982. A new clinical scale for the staging of dementia. Br. J. Psychiatry 140 , $566-572$.

Hutchinson, M., Nakamura, T., Moeller, J.R., Antonini, A., Belakhlef, A., Dhawan, V., Eidelberg, D., 2000. The metabolic topography of essential blepharospasm: a focal dystonia with general implications. Neurology 55 (5), 673-677.

Ishii, K., Sasaki, M., Yamaji, S., Sakamoto, S., Kitagaki, H., Mori, E., 1997. Demonstration of decreased posterior cingulate perfusion in mild Alzheimer's disease by means of $\mathrm{H} 215 \mathrm{O}$ positron emission tomography. Eur. J. Nucl. Med. 24 (6), 670-673.

Johnson, K.A., Jones, K., Holman, B.L., Becker, J.A., Spiers, P.A., Satlin, A., Albert, M.S., 1998. Preclinical prediction of Alzheimer's disease using SPECT. Neurology 50 (6), 1563-1571.

Jueptner, M., Weiller, C., 1995. Review: does measurement of regional cerebral blood flow reflect synaptic activity? Implications for PET and fMRI. NeuroImage 2 (2), 148-156.

Kaplan, E., Goodglass, H., Weintraub, S., 1983. Boston Naming Test Lea \& Febiger, Philadelphia.

Kennedy, A.M., Frackowiak, R.S., Newman, S.K., Bloomfield, P.M., Seaward, J., Roques, P., Lewington, G., Cunningham, V.J., Rossor, M.N., 1995. Deficits in cerebral glucose metabolism demonstrated by positron emission tomography in individuals at risk of familial Alzheimer's disease. Neurosci. Lett. 186 (1), 17-20.

Kim, J.J., Fanselow, M.S., 1992. Modality-specific retrograde amnesia of fear. Science 256 (5057), 675-677.

Leibovitch, F.S., Black, S.E., Caldwell, C.B., McIntosh, A.R., Ehrlich, L.E., Szalai, J.P., 1999. Brain SPECT imaging and left hemispatial neglect covaried using partial least squares: the Sunnybrook Stroke study. Hum. Brain Mapp. 7 (4), 244-253.

Logothetis, N.K., Guggenberger, H., Peled, S., Pauls, J., 1999. Functional imaging of the monkey brain. Nat. Neurosci. 2 (6), 555-562.

Lustig, C., Snyder, A.Z., Bhakta, M., O’Brien, K.C., McAvoy, M., Raichle, M.E., Morris, J.C., Buckner, R.L., 2003. Functional deactivations: change with age and dementia of the Alzheimer type. Proc. Natl. Acad. Sci U. S. A. 100 (24), 14504-14509.

Mattis, S., 1976. Mental status examination for organic mental syndrome in the elderly patient. In: Bellak, L., Karasu, T.B. (Eds.), Geriatr. Psychiatry Grune \& Stratton, New York, NY, pp. 77-121.

McGeer, P.L., Kamo, H., Harrop, R., Li, D.K., Tuokko, H., McGeer, E.G., Adam, M.J., Ammann, W., Beattie, B.L., Calne, D.B., et al., 1986a. Positron emission tomography in patients with clinically diagnosed Alzheimer's disease. CMAJ. 134 (6), 597-607.

McGeer, P.L., Kamo, H., Harrop, R., McGeer, E.G., Martin, W.R., Pate, B.D., Li, D.K., 1986b. Comparison of PET, MRI, and CT with pathology in a proven case of Alzheimer's disease. Neurology 36 (12), 1569-1574.

McGeer, E.G., McGeer, P.L., Harrop, R., Akiyama, H., Kamo, H., 1990a. Correlations of regional postmortem enzyme activities with premortem local glucose metabolic rates in Alzheimer's disease. J. Neurosci. Res. 27 (4), 612-619.

McGeer, E.G., Peppard, R.P., McGeer, P.L., Tuokko, H., Crockett, D., Parks, R., Akiyama, H., Calne, D.B., Beattie, B.L., Harrop, R., 1990b. 18Fluorodeoxyglucose positron emission tomography studies in presumed Alzheimer cases, including 13 serial scans. Can. J. Neurol. Sci. 17 (1), 1-11.

McIntosh, A.R., Gonzalez-Lima, F., 1994. Structural Equation Modeling and its application to network analysis in functional brain mapping. Hum. Brain Mapp. 2 (1-2), 2-22. 
McIntosh, A.R., Bookstein, F.L., Haxby, J.V., Grady, C.L., 1996. Spatial pattern analysis of functional brain images using partial least squares. NeuroImage 3 (3 Pt 1), 143-157.

McKhann, G., Drachman, D., Folstein, M., Katzman, R., Price, D., Stadlan, E.M., 1984. Clinical diagnosis of Alzheimer's disease: report of the NINCDS-ADRDA Work Group under the auspices of Department of Health and Human Services Task Force on Alzheimer's Disease. Neurology 34 (7), 939-944.

Meguro, K., Blaizot, X., Kondoh, Y., Le Mestric, C., Baron, J.C., Chavoix, C., 1999. Neocortical and hippocampal glucose hypometabolism following neurotoxic lesions of the entorhinal and perirhinal cortices in the non-human primate as shown by PET. Implications for Alzheimer's disease. Brain 122 (Pt 8), 1519-1531.

Mielke, R., Schroder, R., Fink, G.R., Kessler, J., Herholz, K., Heiss, W.D., 1996. Regional cerebral glucose metabolism and postmortem pathology in Alzheimer's disease. Acta Neuropathol. 91 (2), 174-179.

Millien, I., Blaizot, X., Giffard, C., Mezenge, F., Insausti, R., Baron, J.C., Chavoix, C., 2002. Brain glucose hypometabolism after perirhinal lesions in baboons: implications for Alzheimer disease and aging. J. Cereb. Blood Flow Metab. 22 (10), 1248-1261.

Minoshima, S., Giordani, B., Berent, S., Frey, K.A., Foster, N.L., Kuhl, D.E., 1997. Metabolic reduction in the posterior cingulate cortex in very early Alzheimer's disease. Ann. Neurol. 42 (1), 85-94.

Moeller, J.R., Strother, S.C., Sidtis, J.J., Rottenberg, D.A., 1987. Scaled subprofile model: a statistical approach to the analysis of functional patterns in positron emission tomographic data. J. Cereb. Blood Flow Metab. 7 (5), 649-658.

Moeller, J.R., Ishikawa, T., Dhawan, V., Spetsieris, P., Mandel, F., Alexander, G.E., Grady, C., Pietrini, P., Eidelberg, D., 1996. The metabolic topography of normal aging. J. Cereb. Blood Flow Metab. 16 (3), $385-398$.

Nakamura, T., Ghilardi, M.F., Mentis, M., Dhawan, V., Fukuda, M., Hacking, A., Moeller, J.R., Ghez, C., Eidelberg, D., 2001. Functional networks in motor sequence learning: abnormal topographies in Parkinson's disease. Hum. Brain Mapp. 12 (1), 42-60.

Nelson, H.E., O'Connell, A., 1978. Dementia: the estimation of premorbid intelligence levels using the New Adult Reading Test. Cortex 14 (2), $234-244$.

Okamura, N., Arai, H., Maruyama, M., Higuchi, M., Matsui, T., Tanji, H., Seki, T., Hirai, H., Chiba, H., Itoh, M., Sasaki, H., 2002. Combined analysis of CSF tau levels and [(123)I] iodoamphetamine SPECT in mild cognitive impairment: implications for a novel predictor of Alzheimer's disease. Am. J. Psychiatry 159 (3), 474-476.

Ouchi, Y., Nobezawa, S., Okada, H., Yoshikawa, E., Futatsubashi, M., Kaneko, M., 1998. Altered glucose metabolism in the hippocampal head in memory impairment. Neurology 51 (1), 136-142.

Petersen, R.C., Stevens, J.C., Ganguli, M., Tangalos, E.G., Cummings, J.L., DeKosky, S.T., 2001. Practice parameter: early detection of dementia: mild cognitive impairment (an evidence-based review): report of the Quality Standards Subcommittee of the American Academy of Neurology. Neurology 56 (9), 1133-1142.

Pietrini, P., Azari, N.P., Grady, C.L., Salerno, J.A., Gonzales-Aviles, A., Heston, L.L., Pettigrew, K.D., Horwitz, B., Haxby, J.V., Schapiro, M.B., 1993. Pattern of cerebral metabolic interactions in a subject with isolated amnesia at risk for Alzheimer's disease: a longitudinal evaluation. Dementia 4 (2), 94-101.

Poline, J.B., Worsley, K.J., Evans, A.C., Friston, K.J., 1997. Combining spatial extent and peak intensity to test for activations in functional imaging. NeuroImage 5 (2), 83-96.

Press, D.Z., Casement, M., Alsop, D., 2003. Hippocampal formation hyperperfusion in early Alzheimer's disease. Neurology 60 (Suppl. 1), A113.

Raichle, M.E., MacLeod, A.M., Snyder, A.Z., Powers, W.J., Gusnard, D.A., Shulman, G.L., 2001. A default mode of brain function. Proc. Natl. Acad. Sci. U. S. A. 98 (2), 676-682.
Rosen, W.G., 1983. Neuropsychological investigation of memory visuoconstructional, visuoperceptional, and language abilities in senile dementia of the Alzheimer type. In: Mayeux, R., Rosen, W.G. (Eds.), The Demenias, vol. 38. Raven Press, New York, pp. 65-75.

Rubin, E., Dhawan, V., Moeller, J.R., Takikawa, S., Labar, D.R., Schaul, N., Barr, W.B., Eidelberg, D., 1995. Cerebral metabolic topography in unilateral temporal lobe epilepsy. Neurology 45 (12), 2212-2223.

Sackeim, H.A., Prohovnik, I., Moeller, J.R., Brown, R.P., Apter, S., Prudic, J., Devanand, D.P., Mukherjee, S., 1990. Regional cerebral blood flow in mood disorders. I. Comparison of major depressives and normal controls at rest. Arch. Gen. Psychiatry 47 (1), 60-70.

Sackeim, H.A., Prohovnik, I., Moeller, J.R., Mayeux, R., Stern, Y., Devanand, D.P., 1993. Regional cerebral blood flow in mood disorders. II. Comparison of major depression and Alzheimer's disease. J. Nucl. Med. 34 (7), 1090-1101.

Salmon, E., Collette, F., Degueldre, C., Lemaire, C., Franck, G., 2000. Voxel-based analysis of confounding effects of age and dementia severity on cerebral metabolism in Alzheimer's disease. Hum. Brain Mapp. 10 (1), 39-48.

Signorini, M., Paulesu, E., Friston, K., Perani, D., Colleluori, A., Lucignani, G., Grassi, F., Bettinardi, V., Frackowiak, R.S., Fazio, F., 1999. Rapid assessment of regional cerebral metabolic abnormalities in single subjects with quantitative and nonquantitative [18F]FDG PET: a clinical validation of statistical parametric mapping. NeuroImage 9 (1), $63-80$.

Silverman, D.H., Small, G.W., Chang, C.Y., Lu, C.S., Kung De Aburto, M.A., Chen, W., Czernin, J., Rapoport, S.I., Pietrini, P., Alexander, G.E., Schapiro, M.B., Jagust, W.J., Hoffman, J.M., Welsh-Bohmer, K.A., Alavi, A., Clark, C.M., Salmon, E., de Leon, M.J., Mielke, R., Cummings, J.L., Kowell, A.P., Gambhir, S.S., Hoh, C.K., Phelps, M.E., 2001. Positron emission tomography in evaluation of dementia: regional brain metabolism and long-term outcome. JAMA 286 (17), $2120-2127$.

Smith, G.S., de Leon, M.J., George, A.E., Kluger, A., Volkow, N.D., McRae, T., Golomb, J., Ferris, S.H., Reisberg, B., Ciaravino, J., et al., 1992. Topography of cross-sectional and longitudinal glucose metabolic deficits in Alzheimer's disease. Pathophysiologic implications. Arch. Neurol. 49 (11), 1142-1150.

Stern, Y., Sano, M., Paulson, J., Mayeux, R., 1987. Modified mini-mental state examination: validity and reliability. Neurology 37 (Suppl. 1), 179.

Stern, Y., Moeller, J.R., Anderson, K.E., Luber, B., Zubin, N.R., DiMauro, A.A., Park, A., Campbell, C.E., Marder, K., Bell, K., Van Heertum, R., Sackeim, H.A., 2000. Different brain networks mediate task performance in normal aging and $\mathrm{AD}$ : defining compensation. Neurology 55 (9), $1291-1297$.

Su, P.C., Ma, Y., Fukuda, M., Mentis, M.J., Tseng, H.M., Yen, R.F., Liu, H.M., Moeller, J.R., Eidelberg, D., 2001. Metabolic changes following subthalamotomy for advanced Parkinson's disease. Ann. Neurol. 50 (4), 514-520.

Tabert, M.H., Albert, S.M., Borukhova-Milov, L., Camacho, Y., Pelton, G., Liu, X., Stern, Y., Devanand, D.P., 2002. Functional deficits in patients with mild cognitive impairment: prediction of AD. Neurology 58 (5), 758-764.

Talairach, J., Tournoux, P., 1988. Co-Planar Stereotaxic Atlas of the Human Brain. Thieme Medical Publishers, Inc., New York.

Vogt, B.A., Finch, D.M., Olson, C.R., 1992. Functional heterogeneity in cingulate cortex: the anterior executive and posterior evaluative regions. Cereb. Cortex 2 (6), 435-443.

Wechsler, D., 1981. Wechler Adult Intelligence Scale Revised. The Psychological Corp., New York.

Zola-Morgan, S.M., Squire, L.R., 1990. The primate hippocampal formation: evidence for a time-limited role in memory storage. Science 250 (4978), 288-290. 